\title{
Performance of Gahver-Stehfest Numerical Laplace Inversion Method on Option Pricing Formulas
}

\author{
Endah R.M. Putri and Sentot Didik Surjanto
}

\begin{abstract}
In this paper we study the performance of GahverStehfest numerical Laplace inversion method. The method is applied to some simple functions which have analytical Laplace inversion and the option pricing formulas which their analytical inversions are not available. The accuracy and efficiency of the methods for each functions are presented.
\end{abstract}

Index Terms-American call option, Laplace transform, numerical inversion, optimal exercise price.

\section{INTRODUCTION}

$\mathbf{L}$ APLACE transform method is a widely known method to obtain analytical solution of a mathematical model consists of a differential equation [1]. Many applications in various fields of the Laplace transform method can be found in literature. The application of Laplace transform method in diffusion and wave problem can be found in [2], [3], [4], [5]. As the solution of financial derivative pricing such as option pricing problem can be obtained via diffussion process [6], then the Laplace transform method is also applied in financial problems.

The use of Laplace transform and its analytic inversion on some simple basic functions are common in literature [1], [7]. However, the widely use of Laplace transform in application, leads to more complicated functions or differential equations. Those equations are often solved in Laplace space but no analytical inversion obtained. Consequently, numerical Laplace inversion methods are applied to get the solution in the original space.

The existing numerical Laplace inversion method such as Gaver-Stehfest method [8], Papoulis-Legendre method [9], Schappery method [10], and Durbin method [9], are commonly used for many applications such as engineering field. GaverStehfest method is known as a relatively simple method to use [9] which becomes our interest to apply on some basic functions and option pricing functions. The method is studied in this paper to present the performance of the method in term of accuracy and efficiency.

First stage presented is the accuracy and efficiency of the methods to some simple basic formulas. The formulas have the analytical solutions which are used to measure the accuracy of the methods. The computational time required to obtain the value in the original space are considered as the efficiency

Manuscript received May 3, 2017; accepted July 14, 2017. The authors thank to the Department of Mathematics, Institut Teknologi Sepuluh Nopember, Surabaya, for the financial support in the form of Local Research Grant 2016.

The authors are with the Department of Mathematics, Institut Teknologi Sepuluh Nopember, Surabaya 60111, Indonesia. Email: endahrmp@matematika.its.ac.id of the methods. As the profile of the performance on the simple formulas obtained, then the method is applied to the pricing formulas of the American call options with similar procedure in observing the accuracy and efficiency. The pricing formulas are obtained based on an analytic approximation of the American put options which used a pseudo-steady state approximation [11].

This paper is organized as follows. Section 2 provides the review of the numerical Laplace inversion used in this paper. Section 3 presents the application of the methods on some simple basic formulas and section 4 presents the application on the optimal exercise price formula of American call options.

\section{GAVER-Stehfest Method}

The Gaver-Stehfest method is often used in engineering fields to solve linear and non-linear diffusion problem. A function in Laplace space, $\hat{F}$, is inverted into the original time $\mathrm{t}$ by the following algorithm:

$$
\left\{\begin{aligned}
f(t) \approx & {\left[\frac{\ln 2}{t}\right] \sum_{n=1}^{N} V_{n} \hat{F}\left(\frac{n \ln 2}{t}\right) } \\
V_{n}=(-1)^{\frac{N}{2}+n} & k^{N / 2}(2 k) ! \\
& \sum_{k=\frac{n+1}{\min (n, N / 2)}}^{n+k) ! k !(n-k) !(2 k-n) !}
\end{aligned}\right.
$$

where $N$ is an even number, $\mathrm{n}$ is an integer, $1 \leq n \leq N$, and $\mathrm{k}$ is the greatest integer less than or equal to $(n+1) / 2 . \mathrm{N}$ being even is required for convergence acceleration.

\section{Performance on Some Basic Formulas}

This section presents numerical implementations of GaverStehfest method (see Equation 1) applied in some basic formulas. The analytic inversion of the formulas can be obtained easily with the basic knowledge of the Laplace transform [1]. The Laplace transform is defined as

$$
\mathscr{L}[f(t)]=F(s)=\int_{0}^{\infty} f(t) e^{-s t} d t
$$

where $f(t)$ is a function such that $f(t)=0$ for $t<0$ and $F(s)$ is the Laplace transform of $f(t)$. The existance of Laplace transform for function $f(t)$ can be read in detail in Duffy [7].

Accordingly, the analytic Laplace inversion is defined as

$$
\mathscr{L}^{-1}[F(s)](t)=f(t)=\frac{1}{2 \pi i} \lim _{T \rightarrow \infty} \int_{\gamma-i T}^{\gamma+i T} F(s) e^{s t} d s
$$

where $\gamma$ is a a real number for the convergence of countour integral. 
Here, we use some basic functions whose analytic inversion can be found easily based on Equation 2 and 3. We determine the basic functions based on their types: linear, quadratic, sinusoidal, logarithmic, and exponential. These functions are used to provide the performance measurement of the numerical Laplace inversion later on. The performance is observed based on the accuracy and computational time spent to obtain the inversion.

The accuracy of the Gaver-Stehfest method is justified using the relative errors. The errors are calculated as the mean absolute errors (MAE) shown by Equation 4 as follows,

$$
M A E=\sum_{i=1}^{n} \frac{\left|\tilde{x}_{i}-x_{i}\right|}{n}
$$

The justification of the accuracy of the method is set up by $5 \%$ tolerance and the error must be less than the tolerance limit prescribed. We use Matlab R2013a and PC specification Intel(R)Core(TM) i5 - 2400S CPU @ 2.50GHz2.50G Hz, 64bit Operating System RAM 4.00 GB.

The ease of this method by only choosing the value of $\mathrm{N}$ should be observed for finding the most reliable $\mathrm{N}$. The simulation is conducted for various value of $\mathrm{N}$ to observe the most suitable range value of $\mathrm{N}$. In theory, the larger value of $\mathrm{N}$ results better value of inversion, but Cheng and Sidauruk [9] suggested that the value ranges between $6-20$. Therefore, we tested the functions by choosing $N=2, \ldots, 22$. The results are shown in Table I and Table II in the Appendix.

The $\gamma$ in function of the fifth coloumn in Table I and II is The Euler-Mascheroni constant which has numerical value $\gamma=0.577215664901532860606512090082402431042 \ldots$ as in [12].

Table I and II show that the Gaver-Stehfest numerical inversion method can provide fast convergent results as the number of $\mathrm{N}$ increases. By comparing the numerical results with the analytical ones, the MAE (Mean Absolute Error) is calculated for every basic functions tested. Figure 1 to 5 show that the MAE decreases significantly as the $\mathrm{N}$ increases. In detail for Fig 1 and 3, the MAEs are smoothly decreasing while the 2,4, and 5 show different behaviour. The decreasing functions of MAE in 2,4, and 5 have different trends, one is smoothly decreasing while the other fluctuate decreasing. The value of MAE which is less than $5 \%$ for all functions after $N=12$. For all basic functions tested, the results show that Gaver-Stehfest method is significantly accurate, efficient, and easy, to use for numerical Laplace inversion.

To continue the used of Gaver-Stehfest in other functions with higher complexity, here we propose the optimal exercise formula of American call options and American down and out call options in the Laplace space. The functions have polynomial forms which is one type of the function tested.

\section{Performance on American Options}

In this section, the equation optimal exercise price of American call options is tested using the numerical Laplace inversion methods. For clarity we present the formulas derived based on [13].

The American call options governing equation based on the Black-Scholes model is similar to European options with the

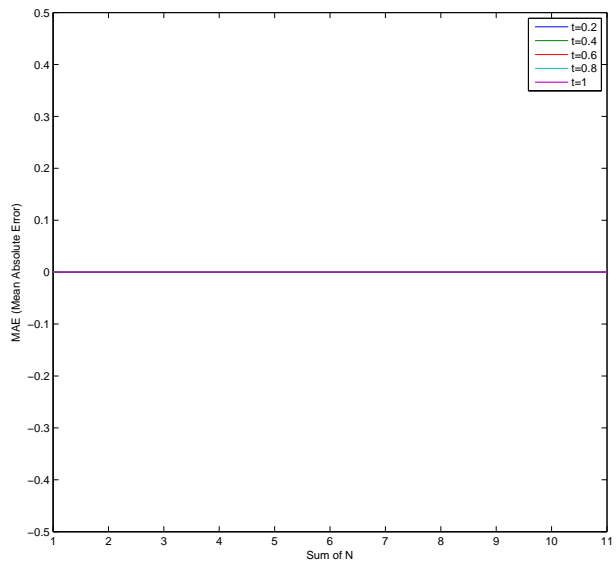

(a)

Fig. 1. The MAE profile for $f(t)=1$ in Table I

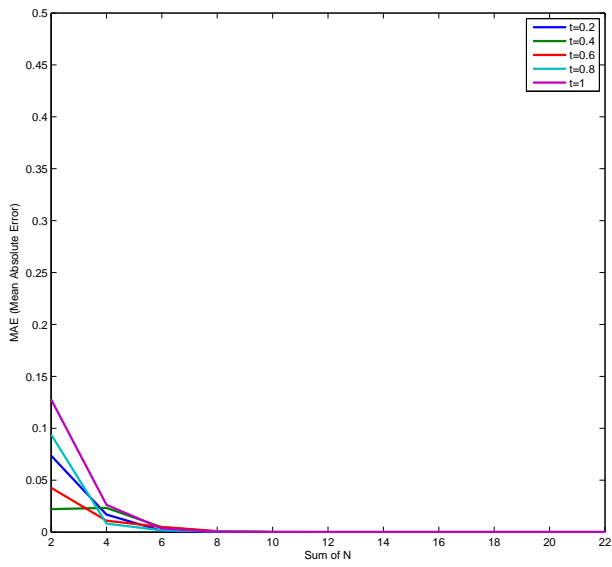

(a)

Fig. 2. The MAE profile for $f(t)=t e^{-t}$ in Table I

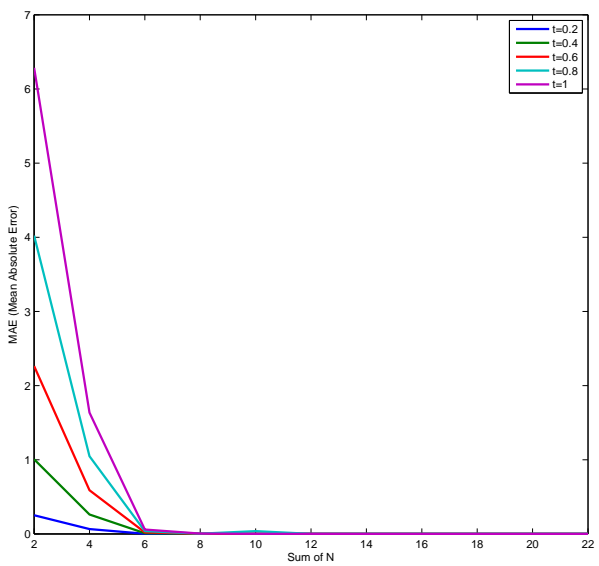

(a)

Fig. 3. The MAE profile for $f(t)=t^{2}$ in Table I 


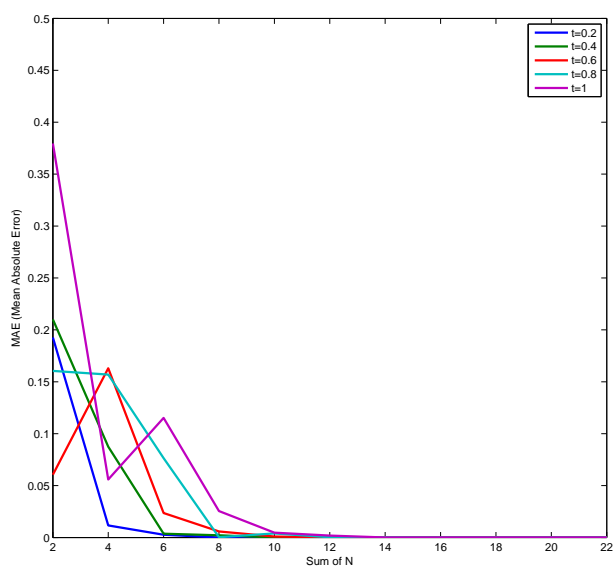

(a)

Fig. 4. The MAE profile for $f(t)=\sin (t)$ in Table I

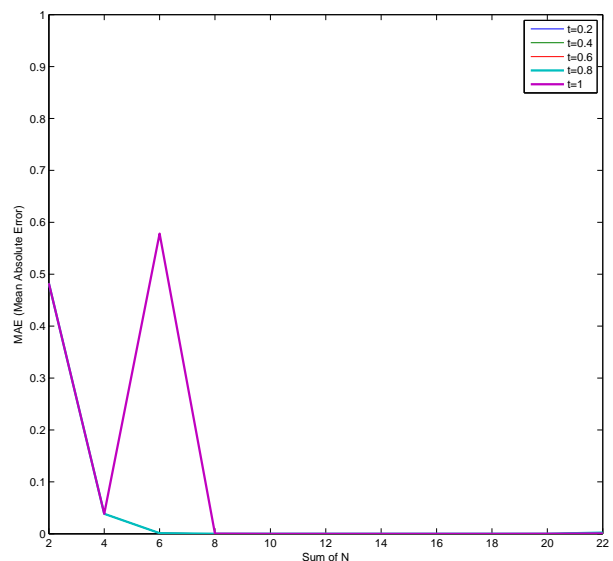

(a)

Fig. 5. The MAE profile for $f(t)=-\gamma-\ln (t))$ in Table I

initial and boundary conditions but the American style has additional conditions as a free boundary conditions. While the European options has the analytic solution, the American option has no analytic solution [11]. The optimal exercise privilege in American options makes the option valuations become more complicated.

The complete PDE system of the dividend-paid American call options with the Black-Scholes equation as the governing equation, can be summarized as follows:

$$
\left\{\begin{array}{l}
\frac{\partial V}{\partial t}+\frac{1}{2} \sigma^{2} S^{2} \frac{\partial^{2} V}{\partial S^{2}}+(r-\delta) S(t) \frac{\partial V}{\partial S}-r V=0 \\
V(0, t)=0 \\
V(S, T)=\max (S-K, 0) \\
V\left(S_{f}(t), t\right)=S_{f}(t)-K \\
\frac{\partial V}{\partial S}\left(S_{f}(t), t\right)=1
\end{array}\right.
$$

where $V$ is the option price, $S$ is the stock price, $S_{f}$ is the optimal exercise price, $K$ is the strike price. The parameter $r$ is the risk-free interest rate, $\sigma$ is the volatility, and $\delta$ is the dividend paid. The two latter boundary conditions in Equation 5 are the optimal exercise boundaries that have been the part of the solution.

Using the solution procedure following Zhu [11], we can obtain the formula to calculate the optimal exercise price in Laplace space with dimensionless variables similar to the ones in Zhang and Zhu [14]. Equation for the optimal exit price is obtained explicitly in Laplace space,

$$
\begin{aligned}
& \hat{X}_{f}^{q_{2}}\left\{\frac{q_{1}(\beta-\alpha)+(p+\alpha)}{q_{1}(p+\alpha)(p+\beta)}\right\} \\
& +\hat{X}_{f}\left\{\frac{\beta\left(1-q_{1}\right)}{q_{1} p^{q_{2}}(p+\beta)}\right\}=-\frac{\alpha}{p^{1+q_{2}}(p+\alpha)}
\end{aligned}
$$

where

$$
\begin{gathered}
q_{1,2}=\frac{1+\beta-\alpha}{2} \pm \sqrt{\left(\frac{1+\beta-\alpha}{2}\right)^{2}+(p+\alpha)} \\
\alpha=\frac{2 r}{\sigma^{2}} \\
\beta=\frac{2 \delta}{\sigma^{2}}
\end{gathered}
$$

Equation 6 is highly non-linear and is difficult to invert back to the original space. This fact is different from the one in Zhu [11] that the formula for non-dividend paid American put options is obtained. The analytical inversion of the formula is available for the typical options but not available if the dividend is assumed to be paid [14]. Therefore, a numerical Laplace inversion method should be considered.

In the following, a simulation study of Gaver-Stehfest numerical Laplace inversion method for the optimal exercise formula (Equation 6) is presented. The value will be compared with the results from binomial method with various grid size.

Firstly, we compare the accuracy of the non-dimensional optimal exercise using the Gahver-Stehfest method for numerical Laplace inversion with the one using binomial method. The parameter $N=8$ is used as suggested in [14]. The results obtained from Gahver-Stehfest method has become closer to the binomial results as the contract duration is longer, see Figure 6, 7, and 8. The perpetual value of both method is in a good agreement as shown in Figure 9.

Secondly, we explore the effect of parameter $\mathrm{N}$ in GahverStehfest method to the computation results. In the previous basic function tested, the error decreases when the $\mathrm{N}$ becomes larger. However, being applied in the optimal exercise price of American call options, larger $\mathrm{N}$ can not guarantee that the accuracy will increase [14]. The facts are shown in Figure 10, 11 , and 12. The figures show that for $N=2,4,8$, the results are stable and close to the binomial's results. For $N=24$, the result is unstable and does not reach convergence. In conclusion, the greater $\mathrm{N}$ does not guarantee the better result as spotted in [14]. Therefore, the use of Gahver-Stehfest method in other formulation needs a pre-study to see the proper $\mathrm{N}$ to be chosen in the calculation. 


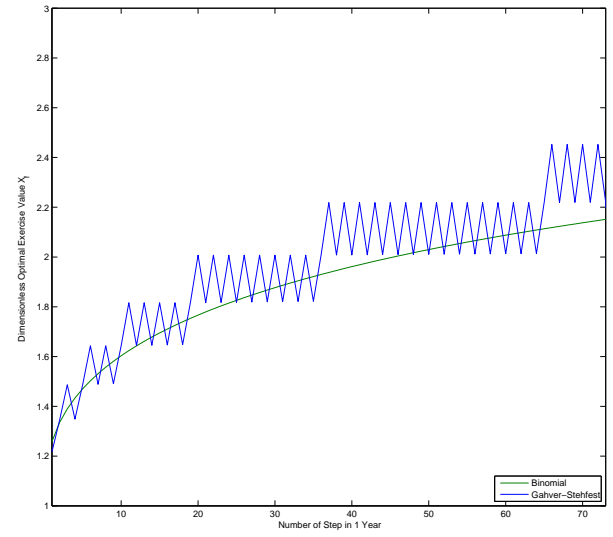

Fig. 6. Dimensionless Optimal Exercise Price $X_{f}$ for $T=1$ year

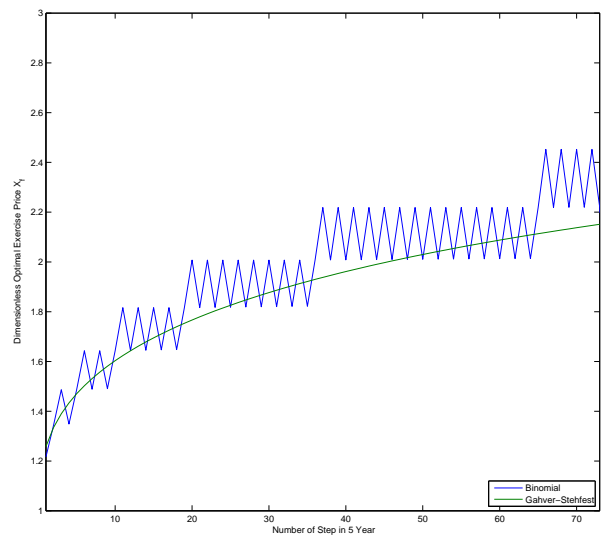

Fig. 7. Dimensionless Optimal Exercise Price $X_{f}$ for $T=5$ year

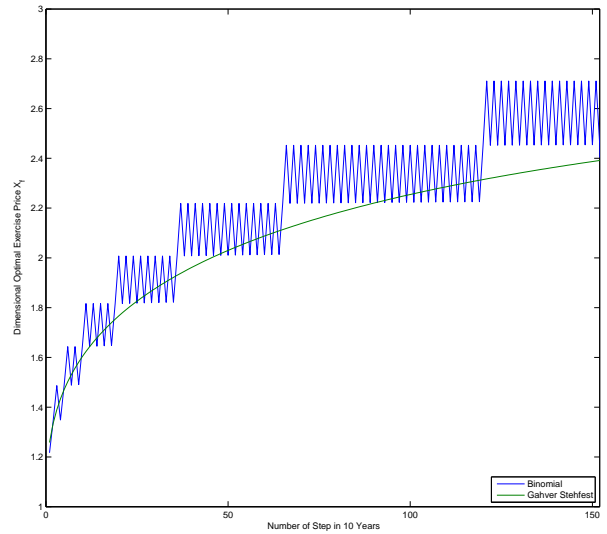

Fig. 8. Dimensionless Optimal Exercise Price $X_{f}$ for $T=10$ year

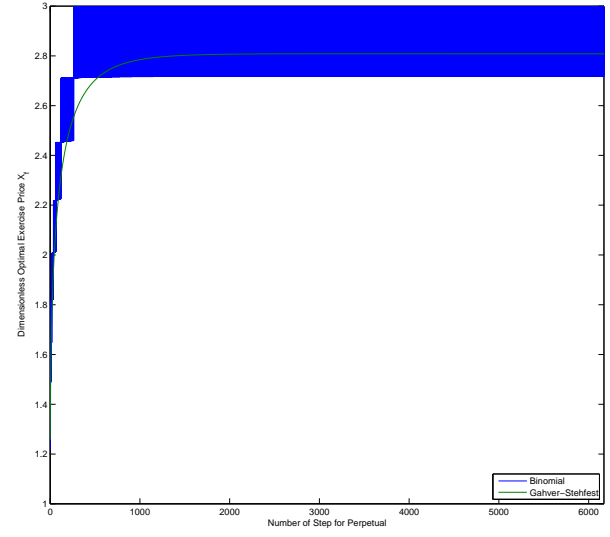

Fig. 9. Dimensionless Optimal Exercise Price $X_{f}$ for Perpetual

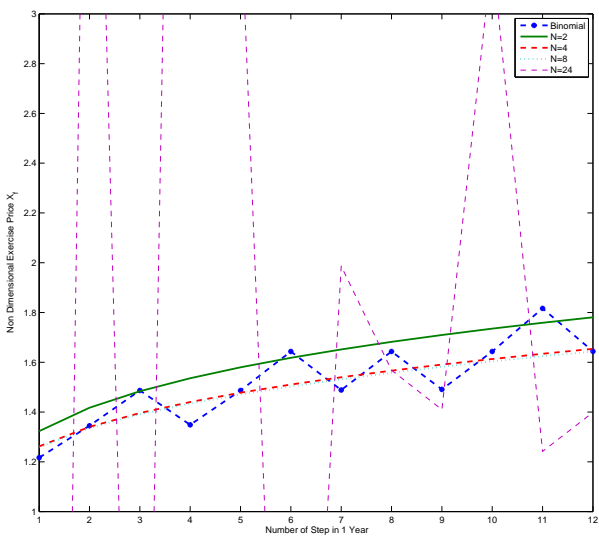

Fig. 10. Comparison of Many $\mathrm{N}$ in Gahver-Stehfest and 1 Year Binomial

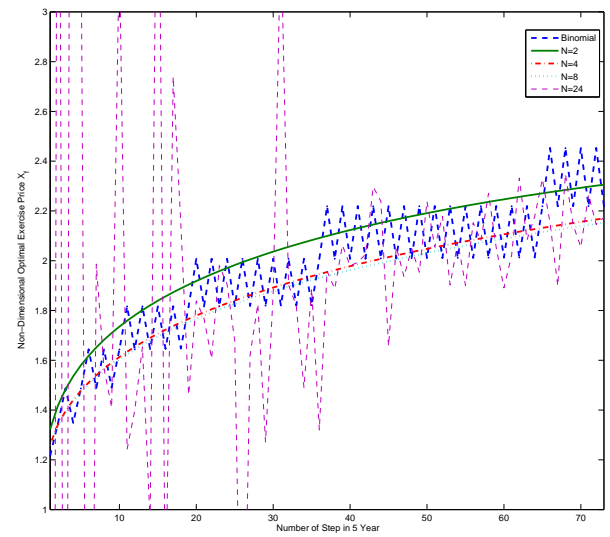

Fig. 11. Comparison of Many $\mathrm{N}$ in Gahver-Stehfest and 5 Year Binomial 


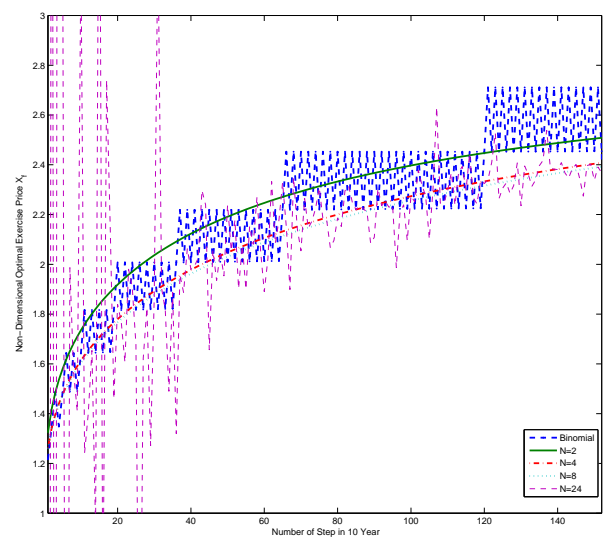

Fig. 12. Comparison of Many $\mathrm{N}$ in Gahver-Stehfest and 10 Year Binomial

\section{CONCLUSIONS}

The use of Gahver-Stehfest method needs a justification of parameter $N$ to obtain good numerical inversion results. For some simple functions, the set of $\mathrm{N}$ is in the range of $N=$ $8, \ldots, 22$. For a more complicated formula which is non-linear, an optimal exercise formula ini Laplace space is presented and simulated. The results suggested that more than $N=22$ will result a non-convergence values.

Comparison between the optimal exercise price resulted from the numerical Laplace inversion Gahver-Stehfest and method binomial method shows that the accuracy is relatively close to the binomial results.

\section{APPENDIX}

TABLE I

Test of GahVer-Stehfest Method on Some Basic Functions

\begin{tabular}{|c|c|c|c|}
\hline \multirow{2}{*}{$\mathrm{N}$} & \multicolumn{3}{|c|}{ Results(Numeric)(Computational Time) } \\
\cline { 2 - 4 } & $\begin{array}{c}f(t)=1, \\
F(s)=1 / s\end{array}$ & $\begin{array}{c}f(t)=e^{-1}, \\
\end{array}$ & $\begin{array}{c}f(t)=\frac{1}{(s+1)^{2}} \\
F(s)=2 ! / s^{3}\end{array}$ \\
\hline 2 & $(1)(0.0453)$ & $(0.2401)(0.0148)$ & $(7.2848)(0.0135)$ \\
\hline 4 & $(1)(0.0098)$ & $(0.3416)(0.0138)$ & $(-0.6360)(0.0155)$ \\
\hline 6 & $(1)(0.0092)$ & $(0.3644)(0.0142)$ & $(1.0586)(0.0168)$ \\
\hline 8 & $(1)(0.0152)$ & $(0.3676)(0.0175)$ & $(1.0042)(0.0147)$ \\
\hline 10 & $(1)(0.0123)$ & $(0.3679)(0.0146)$ & $(0.9999)(0.0128)$ \\
\hline 12 & $(1)(0.0130)$ & $(0.3679)(0.0210)$ & $(0.9999)(0.0137)$ \\
\hline 14 & $(1)(0.0120)$ & $(0.3679)(0.0150)$ & $(1.0000)(0.0232)$ \\
\hline 16 & $(1)(0.0167)$ & $(0.3679)(0.0236)$ & $(1.0000)(0.0249)$ \\
\hline 18 & $(1)(0.0151)$ & $(0.3679)(0.0274)$ & $(1.0000)(0.0257)$ \\
\hline 20 & $(1)(0.0146)$ & $(0.3679)(0.0179)$ & $(1.0000)(0.0179)$ \\
\hline 22 & $(1)(0.0269)$ & $(0.3679)(0.0337)$ & $(1.0000)(0.0195)$ \\
\hline
\end{tabular}

\section{REFERENCES}

[1] J. L. Schiff, The Laplace Transform: Theory and Applications. Springer Science \& Business Media, October 1999

[2] G. Davis, "A Laplace transform technique for the analytical solution of a diffusion- convection equation over a finite domain," Applied Mathematical Modelling, vol. 9, no. 1, pp. 69 - 71, 1985. [Online]. Available: http://www.sciencedirect.com/science/article/pii/0307904X8590143X
TABLE II

Test of Gahver-Stehfest Method on Some Basic Functions (CONTINUATION)

\begin{tabular}{|c|c|c|}
\hline \multirow{2}{*}{$\mathrm{N}$} & \multicolumn{2}{|c|}{ Results(Numeric)(Computational Time) } \\
\cline { 2 - 3 } & $f(t)=\sin (t)$, & $f(t)=-\gamma-\ln (t)$, \\
& $F(s)=\frac{1}{s^{2}+1} \quad t=1$ & $F(s)=\frac{\ln (s)}{s} \quad t=1$ \\
\hline 2 & $(0.4619)(0.0007)$ & $(-1.0597)(0.0114)$ \\
\hline 4 & $(0.8974)(0.0095)$ & $(-0.6156)(0.0116)$ \\
\hline 6 & $(0.9567)(0.0175)$ & $(-0.5783)(0.0117)$ \\
\hline 8 & $(0.8670)(0.0184)$ & $(-0.5770)(0.0123)$ \\
\hline 10 & $(0.8368)(0.0202)$ & $(-0.5772)(0.0130)$ \\
\hline 12 & $(0.8396)(0.0126)$ & $(-0.5772)(0.0145)$ \\
\hline 14 & $(0.8415)(0.0181)$ & $(-0.5772)(0.0193)$ \\
\hline 16 & $(0.8415)(0.0192)$ & $(-0.5772)(0.0179)$ \\
\hline 18 & $(0.8415)(0.0170)$ & $(-0.5772)(0.0175)$ \\
\hline 20 & $(0.8415)(0.0282)$ & $(-0.5771)(0.0220)$ \\
\hline 22 & $(0.8414)(0.0208)$ & $(-0.5765)(0.0179)$ \\
\hline
\end{tabular}

TABLE III

Test of Gahver-Stehfest Method on Optimal Exercise Formula OF VANILla AMERICAN CALl OPTIONS

\begin{tabular}{|c|c|c|c|}
\hline \multirow{2}{*}{$\mathrm{N}$} & \multicolumn{3}{|c|}{ (Results)(Computational Time) } \\
\cline { 2 - 4 } & $t=1$ & $t=5$ & $t=10$ \\
\hline 2 & $(3.4477)(0.0707)$ & $(4.5722)(0.2309)$ & $(4.9256)(0.2667)$ \\
\hline 4 & $(3.1013)(0.0705)$ & $(4.3235)(0.2731)$ & $(4.8019)(0.5423)$ \\
\hline 6 & $(3.0693)(0.0987)$ & $(4.2818)(0.4369)$ & $(4.7840)(0.8070)$ \\
\hline 8 & $(3.0699)(0.1217)$ & $(4.2780)(0.5403)$ & $(4.7836)(1.1783)$ \\
\hline 10 & $(3.0704)(0.2417)$ & $(4.2779)(0.6933)$ & $(4.7839)(1.3350)$ \\
\hline 12 & $(3.0689)(0.1885)$ & $(4.2780)(0.8118)$ & $(4.7840)(1.6518)$ \\
\hline 14 & $(3.0662)(0.2350)$ & $(4.2779)(0.9494)$ & $(4.7840)(1.8690)$ \\
\hline 16 & $(3.0630)(0.2478)$ & $(4.2779)(1.1109)$ & $(4.7840)(2.1643)$ \\
\hline 18 & $(3.0600)(0.2792)$ & $(4.2780)(1.1893)$ & $(4.7840)(2.4023)$ \\
\hline 20 & $(3.0597)(0.2950)$ & $(4.2780)(1.3368)$ & $(4.7839)(2.7016)$ \\
\hline 22 & $(3.0337)(0.3168)$ & $(4.2786)(1.5241)$ & $(4.7880)(2.9780)$ \\
\hline
\end{tabular}

TABLE IV

Test of GahVer-Stehfest Method on Optimal Exercise Formula of VANILla AMERICAN CALl OPTIONS(CONTINUATION)

\begin{tabular}{|c|c|c|}
\hline \multirow{2}{*}{$\mathrm{N}$} & \multicolumn{2}{|c|}{ (Results)(Computational Time) } \\
\cline { 2 - 3 } & $t=20$ & $\begin{array}{c}\text { Analytic Perpetual } \\
\end{array}$ \\
\hline 2 & $(5.1370)(0.5338)$ & $(5.2884)$ \\
\hline 4 & $(5.1119)(1.0502)$ & $(5.2884)$ \\
\hline 6 & $(5.1208)(1.5805)$ & $(5.2884)$ \\
\hline 8 & $(5.1258)(2.0149)$ & $(5.2884)$ \\
\hline 10 & $(5.1270)(2.6231)$ & $(5.2884)$ \\
\hline 12 & $(5.1272)(3.1516)$ & $(5.2884)$ \\
\hline 14 & $(5.1272)(3.7730)$ & $(5.2884)$ \\
\hline 16 & $(5.1272)(4.3943)$ & $(5.2884)$ \\
\hline 18 & $(5.1272)(4.7415)$ & $(5.2884)$ \\
\hline 20 & $(5.1273)(5.2737)$ & $(5.2884)$ \\
\hline 22 & $(5.1297)(5.8376)$ & $(5.2884)$ \\
\hline
\end{tabular}

[3] H. Eltayeb and A. Klman, "A note on solutions of wave, Laplaces and heat equations with convolution terms by using a double Laplace transform," Applied Mathematics Letters, vol. 21, no. 12, pp. 1324 - 1329, 2008. [Online]. Available: http://www.sciencedirect.com/science/article/pii/S0893965908000761

[4] D. Shah and A. K. Parikh, "Solution of one dimensional wave equation using Laplace transform," International Journal of Science and Research, vol. 4, no. 2, pp. 2264-2267, February 2015.

[5] S. A. D. Bahuguna and R. K. Shukla, "Laplace transform method for one-dimensional heat and wave equations with nonlocal conditions," International Journal of Applied Mathematics and Statistics, vol. 16, 
no. M10, pp. -, March 2010.

[6] Y.-K. Kwok, Mathematical Models of Financial Derivatives, 2nd ed. Springer, 2008.

[7] D. G. Duffy, Transform Methods for Solving Partial Differential Equations, 2nd ed. Chapman and Hall/CRC, 2004.

[8] H. Hassanzadeh and M. P. Darvish, "Comparison of different numerical Laplace inversion methods for engineering applications," Applied Mathematics and Computation, vol. 189, no. 2, pp. 1966 - 1981, 2007.

[9] Y. A. A. Cheng, P. Sidauruk, "Approximate inversion of the Laplace transform," The Mathematica Journal, vol. 4, no. 2, pp. 76-81, 1994.

[10] K. L. Kuhlman, "Review of inverse Laplace transform algorithms for Laplace-space numerical approaches," Numerical Algorithms, vol. 63, no. 2, pp. 339-355, 2013.

[11] S.-P. Zhu, "A new analytical approximation formula for the optimal exercise boundary of American put options," International Journal of Theoretical and Applied Finance, vol. 7, no. 9, pp. 1141-1177, 2006.

[12] T. W. Yee, Vector Generalized Linear and Additive Models: With an Implementation in R, ser. Springer Series in Statistics. Springer, 2015.

[13] E. R. M. Putri, "Stock loans valuation," Ph.D. dissertation, School of Mathematics and Applied Statistics University of Wollongong, http://ro.uow.edu.au/theses/4234, 2014.

[14] S.-P. Zhu and J. Zhang, "Using Laplace transform to price American puts," Dynamics of Continuous, Discrete and Impulsive Systems-B: Applications and Algorithms, vol. 19, no. 4, pp. 447-469, 2012. 